\title{
Entre les lignes
}

\section{Enquête sur les nageurs réguliers de la piscine Pontoise de Paris}

Hadrien Riffaut ${ }^{1}$

\begin{abstract}
[Résumé] Cet article est basé sur une enquête menée auprès de nageurs réguliers d'une piscine parisienne. Il décrit, dans un premier temps, les modalités d'inscription du chercheur sur un terrain dans lequel il est lui-même acteur. II montre comment l'ancrage dans la durée et le partage quotidien de l'activité étudiée constituent deux atouts privilégiés de ce «terrain chez soi ». II s'intéresse, dans un second temps, au rapport des nageurs à l'eau en tant que matière. Une eau associée à un imaginaire hautement porteur de sens et à des sensations de bien-être, de portage ou d'enveloppement, recherchés des nageurs. Il explore enfin les motivations des nageurs à pratiquer leur activité. Lesquelles sont souvent centrées autour d'un besoin de compenser un problème physique ou une difficulté existentielle.
\end{abstract}

Mots-clés : natation, nageurs réguliers, rétributions sociales et identitaires, vulnérabilité, ethnographie du proche.

[Abstract] This article is based on an investigation of regular swimmers in a Parisian pool. Firstly, it describes the method of the survey in a field of work where the researcher is also an actor. It shows how long-term and deep-rooted participation, as well as the daily sharing of the swimming activity, are two main assets of this « field work at home ». Secondly, this article focuses on the connection between swimmers and water as an object. Swimmers sought out water that was associated with a strong supportive imagery and with feelings of well-being, portage and inclusion. Finally, this article explores what motivates swimmers to practice their activity, which is often focused on a need to compensate for a physical problem or an existential issue. Keywords: swimming, regular swimmer, social and identity rewards, vulnerability, ethnography of relative.

\footnotetext{
${ }^{1}$ Université Paris Descartes.
} 
"Se baigner c'est peut-être d'abord ressentir une atmosphère, traverser des influences portantes, éprouver un affect »(Vigarello, 1995 : 116).

\section{Introduction}

La piscine constitue, pour nombre de Français, un univers familier et relativement accessible 2 . Pourtant, malgré l'engouement chaque année constaté pour la natation, cette pratique comme les nageurs qui s'y adonnent demeure peu étudiés par les ethnologues. L'essentiel de la littérature disponible sur le sujet provient de travaux réalisés dans le domaine des Sciences et Techniques des Activités Physiques et Sportives (STAPS), lesquels insistent plutôt sur ses dimensions techniques (Chadi, 2012) ou historiques (Terret, 1992). Lorsque les sciences sociales s'y intéressent c'est essentiellement sous le prisme de l'activité sportive (Schirrer, 2007; Souville, 2005). Mais de manière plus générale, aucun travail n'a été engagé sur l'univers d'un bassin et la sociabilité des nageurs en son sein. De ce point de vue, les travaux ethnographiques ayant trait à d'autres disciplines comme la marche (Le Breton, 2000) ou la course à pied (Segalen, 1994) m'ont été particulièrement précieux pour mener à bien ce travail.

Cette recherche a pour terrain d'enquête une piscine parisienne - la piscine Pontoise $^{3}$ - et pour objet d'étude les nageurs réguliers qui la fréquentent. Elle s'intéresse plus spécifiquement aux rétributions sociales et identitaires recherchées par les nageurs à travers l'exercice de leur activité. Le titre de cet article, «Entre les lignes », résume, à lui seul, la perspective adoptée: comprendre, au-delà de l'activité physique stricto sensu, de ce qui est visible en surface et de ce que les nageurs donnent à voir, ce qui se joue dans un bassin. Plusieurs questions guident cette réflexion. Comment d'un point de vue méthodologique s'est négociée ma place sur ce terrain sachant que je suis, comme mes interlocuteurs, nageur régulier dans le bassin étudié ? Quels sont, du point de vue des nageurs, les enjeux et les motivations qui les poussent à fréquen-

\footnotetext{
2 Selon l'INSEE, le vélo, la natation et la marche sont les pratiques sportives les plus répandues chez les personnes de plus de quinze ans. Enquête «participation culturelle et sportive », mai 2003, INSEE. Concernant l'accessibilité des infrastructures aquatiques, plus de la moitié des Français ( $53 \%$ ) habite à moins de 5 minutes d'une commune équipée d'une piscine couverte. «État des lieux des bassins de natation en France : une approche par les territoires et par les usagers », Ministère chargé des sports, 2009.

${ }^{3}$ La piscine Pontoise compte parmi les infrastructures aquatiques de la capitale répertoriées à l'inventaire des monuments historiques : elle est réputée pour son décor Art déco. Située au cœur du Quartier Latin, la population qui la fréquente est plutôt aisée et majoritairement composée de riverains, d'étudiants et de professionnels exerçant leur activité à proximité.
} 
ter le lieu ? Que viennent-ils y chercher ? Comment cette pratique s'articule-t-elle avec les autres sphères de leur vie sociale?

\section{Temps et quotidienneté : deux atouts privilégiés du « terrain chez-soi "}

Avant de présenter les résultats de ce travail de recherche, il me paraît important de préciser mon appartenance et ma position de «membre » au sein du milieu étudié. À l'instar de la posture adoptée par Loïc Wacquant dans le gym de Woodlawn, ce terrain fut abordé de manière assez « opportuniste » (Wacquant, 2001: 12). Pratiquant la natation depuis plus de quinze ans et quotidiennement dans le bassin étudié depuis sept ans, c'est d'abord la familiarité du lieu puis les premiers constats et découvertes, qui m’ont incité, début 2013, à prendre mes premières notes sur ce que j’observais. Comme la plupart des nageurs étudiés, je nage de manière assez ritualiste : toujours sur le même créneau horaire - de 12 h15 à 13h30, celui-ci étant parfois décalé dans l'après-midi entre $16 \mathrm{~h} 30$ et $18 \mathrm{~h}$. La distance parcourue est rigoureusement la même trois kilomètres -, et respecte un programme précis: six cent soixante mètres de brasse pour s'échauffer, suivi de sept cent quatre-vingt-douze mètres de dos crawlé et mille cinq cent quatre-vingt quarts mètres de crawl pour clore l'entrainement. Mes séances sont autant de rendez-vous qui battent la cadence de mon quotidien. La régularité et l'intensité de la pratique sont, comme nous le verrons, des dispositions partagées avec l'ensemble des nageurs rencontrés. L'ancrage dans la durée, dans un espace circonscrit et composé d'une population bien définie ${ }^{4}$ constituait un terreau favorable à la réalisation de cette entreprise. J'ai commencé, dans un premier temps, par restituer, dans un carnet, le contenu de mes observations sur un mode très fragmentaire puis, dans un second temps, de manière plus appuyée et systématique. Je consignais les anecdotes, les comportements, les interactions ou les régularités observées chez certains nageurs. Il pouvait s'agir des pratiques répétées de chacun comme le dépôt méthodique du sac à un endroit précis, le choix d'un même numéro de cabine, la préférence d'une douche en particulier ou la réalisation d'un programme de natation. L'appropriation du territoire et les habitudes des nageurs, dans le bassin comme sur ses abords, ont constitué les premiers thèmes de mon investigation.

\footnotetext{
${ }^{4}$ La population observée et interrogée dans cette enquête nage majoritairement sur le créneau horaire de midi. Elle compte une répartition égale d'hommes et de femmes entre 25 et 75 ans. II s'agit plutôt de personnes bénéficiant d'une relative autonomie dans leur emploi du temps, exerçant soit une activité libérale (artiste peintre, photographe, musicien), soit de cadre du privé ou de fonctionnaire de catégorie A (ingénieur de recherche, universitaire, enseignant).
} 
Concernant les modalités de mon inscription sur le terrain, la nécessité d'initier l'enquête à couvert s'est imposée à moi. Il est évident que je n'aurais pu bénéficier de la confiance et de la collaboration des autres nageurs si j'étais entré dans le bassin, dans le but explicite de les étudier. Mais cette posture renvoie aussi à des motifs plus personnels. N'appréhendant plus ce lieu en simple usager mais avec l'intention de l'étudier, j'ai vite ressenti le besoin de maintenir une certaine distance pour préserver ce moment de nage privilégié. Ce projet, articulant deux dispositions parfois difficilement conciliables - nager pleinement et enquêter le milieu - m'a conduit à quelques arbitrages méthodologiques. La "participation observante $~_{5}^{5}$ s'est avérée le meilleur compromis pour engager l'investigation. Elle me permettait d'enquêter tout en limitant mon degré d'implication sur le terrain. L'une des difficultés rencontrées sur ce « terrain chez-soi » fut de faire coexister les statuts de nageur et de chercheur qui mécaniquement s'excluent l'un l'autre. En effet, lorsque je nage, il m'est difficile d'enquêter - et surtout d'entrer en interaction avec d'autres nageurs - et lorsque j'enquête, le temps dévolu à la natation se réduit. Mais cette mise à distance volontaire a rapidement représenté un frein. N'ayant accès ni aux discours ni aux représentations des autres nageurs ${ }^{6}$, il m'était difficile de comprendre en profondeur les logiques qui prévalent à leur présence au bassin. Ainsi l'échange sur le mode informel s'est rapidement imposé comme une nécessité. Initialement limités à quelques marques d'inter-reconnaissances -brèves salutations, hochements de tête soulignés d'un sourire - elles se sont avérées plus appuyées ensuite, parfois suivies de courtes conversations. Ces civilités m'ont permis de faire, dans un temps très limité, la connaissance de la quasi-totalité des nageurs réguliers présents sur ce créneau horaire.

Outre mes observations, j'avais désormais accès à certains éléments de leur parcours glanés au gré de mes rencontres. Toutefois, la posture d'enquête à couvert, réduisant ces entrevues à leur seule dimension informelle, n'offrait que des informations parcellaires et fragmentées qu'il me fallait recomposer. Est donc venu le moment de se découvrir et d'annoncer clairement l'objet de ma recherche. La perspective de se voir enquêté, fut, à ma grande surprise, plutôt bien accueillie par mes interlocuteurs. Loin d'y voir une quelconque intrusion, ils se montrèrent curieux et coopératifs. C'est donc de manière graduelle que s'est construite ma place sur ce ter-

\footnotetext{
${ }^{5}$ Entendue comme l'utilisation d'un rôle existant - celui de nageur - pour engager une recherche dans un environnement qui s'avère familier à soi, soit au sens où l'envisage notamment Dominique Schnapper (2010) dans son enquête intitulée « une sociologue au Conseil Constitutionnel ».

${ }^{6}$ Notons que la posture de mise en retrait, de «repli dans sa bulle » est, comme nous le verrons dans la troisième partie, une attitude fréquemment observée chez nombre de nageurs réguliers. Elle répond à une logique d'optimisation du temps de nage qui, sur des créneaux horaires réduits comme le nôtre - 12h15/13h30 - sont précieux.
} 
rain. Il s'est, en effet, écoulé plus d'une année entre l'amorce des premières conversations et l'annonce officielle de mon enquête.

Sans cette présence régulière et dans la durée, je n’aurais jamais pu initier ce travail. En effet, j'ai toujours pensé que le temps et la durée constituaient deux atouts de poids sur lesquels je pouvais m’appuyer. Comme le précise Olivier Schwartz dans la postface du Hobo de Nels Anderson: «le temps et la quotidienneté sont deux agents puissants de banalisation de l'ethnographe et cette banalisation est heureuse car elle est ce qui lui permet d'enquêter»(Schwartz, 2011: 278). Ces deux dimensions ont structuré mon protocole d'enquête. Lequel renvoie à une longue période d'observation des conduites et des comportements, suivie d'entretiens informels menés auprès d'une quinzaine de nageurs dans des contextes et des situations variés. Il pouvait s'agir d'entretiens individuels, parfois de discussions collectives ayant lieu soit dans les douches ${ }^{7}$ ou près des cabines, soit dans les zones de déchaussage ou aux abords de l'établissement, au début ou à l'issue de nos séances. Dans la mesure où ce travail tente, en partie, de comprendre la place que la natation occupe dans l'existence des nageurs réguliers, les sujets abordés ne se résument pas à la seule pratique de la natation. Comme le soulignent François de Singly et Danilo Martuccelli (2009) qui plaident pour une lecture de l'individu transversale et globale, d'autres dimensions de la vie sociale des nageurs sont ainsi explorées ${ }^{8}$. Précisons que certains propos recueillis et mentionnés dans cet article proviennent d'échanges par mails? C'est la raison pour laquelle une partie des citations qui vont suivre relèvent plus du langage écrit que du style conversationnel.

\section{Créer du lien dans le bassin}

Penser la piscine comme un espace de sociabilité ne va, a priori, pas de soi dans la mesure où un bassin donne plutôt à voir un agrégat d'individus qu'un groupe constitué. Ainsi, comment le dialogue avec les autres nageurs a-t-il pu être engagé ? Au sein de quels espaces ? Et dans quelles conditions?

Activité individuelle et solitaire, la natation apparaît peu propice à l'établissement de relations entre nageurs. Plusieurs facteurs expliquent cette disposition. Tout

\footnotetext{
${ }^{7}$ Ici, exclusivement avec des nageurs car les douches ne sont pas mixtes.

${ }^{8}$ Ainsi, nos entrevues portent régulièrement sur des domaines de leur vie quotidienne comme la famille, le travail, les loisirs, la religion, le rapport au corps qui, en écho à la notion employée par Gérard Althabe (1990), permettent de sortir d'un « contexte de communication » qui se réduirait uniquement à la natation et à sa pratique.

${ }^{9}$ Le temps dédié aux échanges avec mes interlocuteurs étant relativement restreint, le recours au mail a constitué un moyen efficace pour approfondir certains points abordés lors de nos conversations.
} 
d'abord la pudeur induite par la quasi nudité des corps placés dans un rapport proxémique encourage l'adoption de postures de replis, peu propices à l'échange. Par ailleurs, si la température de l'eau convient aux corps en exercice, elle s'avère moins adaptée à l'immobilité requise pour engager la conversation. S'ajoute à cela, le port d'un équipement peu avantageux et plus ou moins élaboré (bonnet de bain, lunettes, palmes, plaquettes, etc.) qui incite plus au retrait qu'au dialogue. Ainsi, les rapports de séduction dans un bassin relèvent plus de l'imaginaire que de la réalité observée. L'uniformisation des corps, par le port de la tenue de bain réglementaire, tend aussi à uniformiser les rapports sociaux de sexe, réduisant l'influence du genre dans la relation d'enquête ${ }^{10}$.

Malgré ces critères peu engageants, la piscine abrite pourtant une sociabilité intense. Observée surtout chez les habitués elle survient généralement après un temps relativement long d'adaptation et d'observation réciproque. Le témoignage de Benja$\min ^{11}$ (38 ans, universitaire) à propos d'Elina (50 ans, ingénieure) comme celui de Mounira (la quarantaine, décoratrice de théâtre) à mon égard rendent compte de cette inclination. Benjamin: « je me souviens de toi il y a quelques années, je te voyais passer avec ton casque de vélo, à cette époque tu ne parlais à personne »; Mounira : "ça fait longtemps qu'on se croise et qu'on se salue à peine, j'espère que vous n'y voyez pas de mépris de ma part ! ». Une fois créée, la relation est aussi subtile à entretenir. Les espaces comme les temporalités sont soumis à un protocole obéissant à des règles fines qui échappent aux pratiquants plus occasionnels. Il est, en effet, d'usage d'éviter de saluer un nageur en exercice, le laissant ainsi tout à son mouvement, d'entamer une conversation avec un autre ayant pris du retard sur son entrainement ou d'échanger trop longtemps en bout de ligne soit pour se préserver du refroidissement soit pour s'épargner les commentaires fraternels d'autres nageurs du type : «ça discute, ça discute ! ». Si l'espace du bassin n'est pas véritablement celui de la sociabilité, ses abords le sont davantage. On échange plus volontiers sur les plages, mais rapidement, avant l'évacuation du bassin ${ }^{12}$, plus longuement dans les douches - uniquement entre hommes ou femmes - et surtout à proximité des cabines, une fois rhabillé où le soin affiché et mixte des corps est de mise (coiffure, onction de crème hydratante, maquillage, etc.). Cela dit l'espace qui invite plus directement à l'échange est la zone de déchaussage. Activité partagée par l'ensemble des usagers, elle clôt vérita-

\footnotetext{
${ }^{10}$ L'érotisation des corps est ainsi neutralisée par l'habitude des nageurs de se voir quasi nus au bassin. Si, à l'extérieur, le corps visible relève du domaine de l'intimité, il est à la piscine soumis à une plus grande banalité.

${ }^{11}$ Afin de respecter l'anonymat des personnes enquêtées et la confidentialité des propos recueillis, les prénoms cités dans cet article ont été modifiés.

12 Observation valable uniquement sur les créneaux du matin et du midi qui laissent la place aux groupes scolaires.
} 
blement la séance et appelle la conversation, laquelle peut se poursuivre aux abords de l'établissement ou sur le trajet du retour. Les zones d'échange sont multiples et dans chacune d'entre elles prédomine un type particulier d'interaction. Les conversations collectives sont plutôt engagées dans la zone de déchaussage, près des cabines ou sur le seuil de l'établissement à la différence des entrevues individuelles, surtout initiées dans les douches ou sur le trajet du retour. Les sujets sont quant à eux variables. Si en groupe les commentaires sur la vie de la piscine, les autres nageurs ou les techniques de nage l'emportent, le face-à-face invite davantage à évoquer sa vie personnelle qu'elle soit professionnelle, familiale ou intime. Bien que la proximité mutuelle des corps quasi nus n'augure pas nécessairement l'établissement de rapports de séduction, elle encourage, en revanche, l'instauration d'un lien privilégié, propice aux confidences. Comme le souligne Martine Segalen à propos de la douche d'après course où nudité et intimité sont intimement liées : « les corps se montrent, les masques s'abaissent dans le respect mutuel » (Segalen 1994 : 15).

\section{Des nageurs dans leur élément}

Après avoir abordé la question de mon inscription sur le terrain et traité des formes de sociabilité dans le bassin, interrogeons à présent le rapport des nageurs à l'eau. Comment les nageurs en parlent-ils ? Que viennent-ils y chercher ? Qu'éprouvent-ils à son contact? Comment justifient-ils leur choix de pratiquer cette activité plutôt qu'une autre?

\subsection{La piscine : le lieu où la gravité est moindre}

Quand on les interroge sur leur rapport à l'eau, les nageurs expliquent d'abord l'apprécier pour ses propriétés physiques et son action sur les corps. Sans toutefois le formuler ainsi, c'est bien d'Archimède et de son principe auxquels ils se réfèrent pour évoquer la sensation de bien-être éprouvé dans le bassin. L'environnement sensoriel de l'immersion dans l'eau réduisant l'acuité des sens ${ }^{13}$ contribue au plaisir décrit par les nageurs à son contact. Le récit d'Elina témoigne de cette légèreté recherchée : « au tout début de ma séance, dit-elle, je ressens un réel plaisir à me laisser glisser dans l'eau [...] et me libérer de la pesanteur ». Olivier (la cinquantaine, cadre supérieur) qui revient souvent dans les douches sur les quelques kilos en trop qu'il souhaite perdre, perçoit l'eau comme: « une bonne camarade [...] on peut ressentir avec bonheur que

\footnotetext{
${ }^{13}$ À l'exception du toucher qui est particulièrement stimulé par le frottement de l'eau sur la surface du corps.
} 
notre poids est divisé par sept, ce qui me conduit enfin à un chiffre raisonnable ». Quant à Benjamin, l'eau est pour lui synonyme de liberté du corps d'abord et de souplesse des mouvements ensuite: « j'aime les choses que le corps peut faire dans l'eau, avec souplesse, avec liberté, comme s'il perdait sa matérialité ordinaire $»^{14}$. Si l'eau est certes un élément porteur, qui allège les corps c'est aussi, pour Benjamin, une matière enveloppante à portée hautement régressive. Un imaginaire qu'il associe aux réminiscences du ventre maternel et qui contribue à renforcer la sensation de liberté évoquée précédemment :

Rétrospectivement je peux trouver des raisons à l'évidence de ce rapport. S'y joue quelque chose de très profond, peut-être d'archaïque ou de premier, une régression vers un état d'avant la naissance, à des sensations dont mon corps seul a sans doute gardé la mémoire et dont je n'ai pas de souvenirs conscients.

Mais l'eau qui enveloppe le nageur a d'autres qualités et notamment celle de lui faire prendre conscience de l'unité de son corps. Les termes « glisser », « caresser », « sentir » sont couramment employés pour évoquer le passage de l'eau sur la peau. C'est parce qu'elle agit sur toute la surface du corps, avec plus ou moins de résistance selon la nage pratiquée, que l'eau rend possible la sensation éprouvée d'un corps unifié.

Parmi les vertus de l'eau attribuées par les nageurs, sont également mises en avant ses propriétés apaisantes. Certains comparent leur séance de natation à un exercice de méditation. Yann ( 45 ans, musicien) évoque ainsi sa perception de l'entrainement : « la natation c'est mon yoga, le moyen que j'ai trouvé pour faire le vide et me concentrer sur la nage, les mouvements, le rythme, la glisse, le nombre de longueur ». Dans une perspective similaire, Axel (la trentaine, universitaire) entrevoit sa séance comme un temps de relâchement : « la natation c'est certes l'effort physique, mais c'est certainement plus le lieu d'un équilibre, d'un apaisement, d'une tranquillité ». Ce calme intérieur, cette sensation de vide éprouvée est rendu possible par l'environnement sensoriel de l'immersion dans l'eau : diminution de l'ouie, restriction du champ de vision, olfaction et gout limité par les effluves chloroformés et perception accrue du toucher. Yann rend particulièrement compte de ce phénomène : «le champ de vision est très restreint et rapidement il ne reste que les sensations physiques ».

Ainsi, la légèreté éprouvée dans l'eau et la sensation de portage, d'enveloppement et d'isolement ressentie à son contact constituent autant d'éléments recherchés par les nageurs au cours de leurs séances.

\footnotetext{
${ }^{14}$ Propos issus d'un échange par mail dans lequel je demandais à Benjamin de me préciser ce qu'il ressentait au contact de l'eau.
} 


\subsection{Quand la bulle éclate}

Si les discours des nageurs sur leur pratique sont souvent stylisés, magnifiés, voire spiritualisés, l'expérience quotidienne du bassin rend compte d'une réalité différente. Sans remettre en cause la véracité de leurs propos, il importe de distinguer la production du discours sur la pratique de l'expérience vécue in situ. À la différence de la course à pied, qui offre à ses adeptes une totale liberté de mouvement, la natation est un sport d'intérieur, circonscrit dans l'espace. Au « coureur libre » (Segalen, 1994: 235) peut s'opposer le nageur de bassin contraint de partager un territoire commun. Si l'affluence est particulièrement redoutée des nageurs elle fait pourtant partie de leur quotidien. Nombreux sont les propos recueillis renvoyant à l'encombrement des lignes: "c'est l'autoroute aux heures de pointe aujourd'hui»; "on n'est pas tout seul!». Nager dans une ligne embouteillée expose le nageur aux incidents, chocs, coups de pieds involontaires qui rendent sa séance parfois pénible. L'analogie au trafic autoroutier est particulièrement significative lors des dépassements qui supposent d'anticiper le nageur venu d'en face, de se positionner au centre de la ligne, pour se rabattre aussitôt l'obstacle franchi. La vélocité des uns devient alors peu compatible avec la lenteur des autres. Parmi ces désagréments, ajoutons les styles de nage désarticulés, les battements de pieds prononcés, les refus de dépassement, comme les difficultés de spatialisation de certains nageurs qui entravent les mouvements et réduisent la visibilité des autres. La ligne se charge alors d'une électricité menant parfois à l'altercation et aux invectives. Au-delà de ces perturbations extérieures, des motifs endogènes peuvent aussi entraver le bon déroulement d'une séance comme la fatigue, l'anxiété ou les contrariétés de la vie courante. Alors, l'eau se fait «lourde» et il devient difficile de se mouvoir et d'avancer. La bulle protectrice et enveloppante à l'intérieur de laquelle les nageurs viennent se réfugier éclate en réalité plus souvent qu'ils ne le disent et ce, malgré les discours sublimés, formulés hors du bassin.

\section{Motivations et enjeux des nageurs réguliers à fréquenter le bassin}

Après avoir décrit la relation des nageurs à l'eau rendant compte de la dimension proxémique et spatiale liée à l'acte de nager en piscine, intéressons-nous, à présent, aux motivations avancées par les nageurs pour justifier leur venue régulière au bassin ? Quels sont les enjeux qui prévalent à leur présence en ce lieu ?

\subsection{Un espace de compensation}

Outre l'entretien de leur condition physique, la natation revêt, chez les nageurs, d'autres enjeux parfois très éloignés des seules préoccupations physiques et sportives. 
Parmi elles, on retrouve le besoin d'alléger le corps comme l'esprit, de surmonter des épreuves existentielles ou de réparer une identité blessée ou fragilisée. Les travaux de Jean Christophe Seznec (2011) qui entrevoient un fond dépressif chez les individus prédisposés au sport qu'ils compensent en se créant une identité positive et socialement valorisée peuvent servir de support à la réflexion sur la dimension compensatrice de la natation proposée ici. Ainsi, loin des représentations communément admises du sportif sain de corps et d'esprit, j’ai compris - à travers mon expérience de nageur comme celle de mes interlocuteurs - que le bassin n'était pas seulement le lieu où l'on nage et l'on fait du sport mais qu'il était aussi appréhendé comme « un espace de compensation ».

\subsection{L'eau et les problèmes}

Le titre de cette section renvoie à une conversation au cours de laquelle Nadine (la quarantaine, enseignante) - une habituée préférant les exercices d'aquagym aux allers et retours dans les lignes - appréhendait la piscine comme un lieu propice à alléger les vicissitudes de la vie. Elle souligne : «si je pouvais savoir tout ce que les gens laissent dans cette eau comme problèmes, je serais surprise !»

Si certains nagent pour se délester d'un poids, d'autres parcourent les lignes pour compenser un manque ou résoudre une difficulté. Il peut s'agir de soulager une affection physique comme un problème de dos ou de surpoids, de lutter contre le vieillissement ou de se libérer d'une addiction (cigarette, alcool, nourriture, etc.). Boris (la trentaine, profession non renseignée), ancien obèse, qui nage deux fois par jour - midi et soir - en parcourant une distance excédant les trois kilomètres, s'est bâti une carrure d'athlète digne des nageurs de compétition reléguant ainsi au passé sa silhouette corpulente. Zora (la quarantaine, sans profession) se rend, quant à elle, quotidiennement au bassin pour tenter désespérément de réduire à coups de palmes une " culotte de cheval » $^{15}$ dont elle ne parvient pas à se défaire et qui, lorsque l'on se croise dans une des lignes me réitère avec humour : «l'objectif! Je vais l'atteindre mon objectif !».

À l'intersection des problématiques physiques et existentielles se trouvent les témoignages de Séverine (la trentaine, agent des domaines nationaux) et de Benjamin pour lesquels la pratique régulière de la natation coïncide avec un épisode anorexique. La première raconte que c'est au moment d'entamer son processus de guérison qu'elle a ressenti le besoin de nager : « quand j’allais à la piscine je me sentais lé-

${ }^{15}$ Une culotte de cheval est une augmentation du volume des cuisses, du bassin et des fesses, due à une accumulation de graisse à ce niveau. 
gère, comme lorsque je me privais de nourriture [...] je retrouvais cette sensation $»^{16}$. Le second, quant à lui, s'est mis à nager après le décès de son grand-père et les troubles anorexiques qui suivirent. J'apprendrai plus tard dans un échange de mails, à propos duquel je lui faisais part de mon rapport personnel à l'eau, que pour Benjamin la natation est aussi : " un substitut ou un prolongement pour un sport, un art, une discipline, une carrière - je ne sais pas quel mot choisir - que je n'ai pas faite. Mais c'est une autre histoire ». Par ailleurs, certains peuvent avoir commencé la natation pour soulager une douleur physique et s'y trouver aujourd'hui pour des motifs plus personnels. En témoignent les propos de Jean (la quarantaine, journaliste) : «j'ai commencé à aller à la piscine pour guérir une hernie discale, j’ai continué à y aller pour puiser littéralement l'inspiration pour écrire mon deuxième roman et j’y vais désormais trois fois par semaine parce que c'est devenu addictif ».

À la résolution de ces problèmes physiques s'agrègent d'autres préoccupations d'ordre existentiel. Dans ce cas, nager permet de réparer une biographie plus ou moins accidentée, troublée ou en souffrance. Si la littérature sociologique sur le sujet demeure relativement pauvre, les références littéraires (Barbero, 2016; Pérez Azaústre, 2015 ; Vivès 2008; Ogawa, 2000), poétiques (Cornière, 2015) ou cinématographiques ${ }^{17}$ existantes sont précieuses pour qui souhaite comprendre ce qui se joue dans un bassin. Celles-ci convergent toutes vers la figure d'un nageur fragile et vulnérable. Nager renvoie ainsi à lutter contre l'entropie, contre le désordre d'une existence agitée ou en questionnement. Les poèmes de François de Cornière rendent ainsi compte de la fonction réparatrice de la natation. Peu de temps après le décès de sa compagne survenu des suites d'une longue maladie, l'auteur renoue avec un rituel partagé avec celle qui l'a quitté. Nager c'est alors « respirer » de nouveau, mais surtout oublier : "l'oubli de qui on est : être soi-même l'oubli de qui on a aimé " (Cornière, 2015 : 19). La bouée qu'il a pour habitude d'atteindre et de dépasser est ainsi nommée : «Cap de l'oubli passager» (Cornière, 2015 : 45). Comme Nadine, l'auteur entrevoit la natation comme un moyen de s'affranchir des charges qui pèsent sur son existence: « nager vers la plage en retour comme poussé par un souffle et libéré d'un poids. Absolument. » (Cornière, 2015 : 52). La recherche de l'oubli est aussi mentionnée par Elina pour justifier sa venue quotidienne au bassin. Cette parenthèse qui rythme son quotidien lui permet d'oublier les troubles psychologiques d'une de ses filles, admise en institution, d'oublier les tensions conjugales récurrentes comme les renoncements à une carrière professionnelle plus prometteuse du fait de s'être prioritairement occupée de ses cinq enfants : «j'avance dans l'eau et je laisse derrière moi les pensées para-

\footnotetext{
${ }^{16}$ Propos issus d'une conversation informelle menée aux abords de l'établissement.

${ }^{17}$ Vigo, (1931), Taris, roi de l'eau; Kieslowski, (1993), Trois couleurs : bleu; Chen (2006), Agua, Anspach (2016), L'effet aquatique.
} 
sites, les frustrations, les déceptions et les chagrins ». Pour Axel, nager lui permet : « de compenser l'activité mentale ». Olivier emploie quant à lui la notion « d'exutoire » : « je me sers de l'eau comme un exutoire, j'y laisse du gras, de la mauvaise humeur et je retrouve un souffle et un bien-être évident ».

Dans un autre registre, les romans de Yoko Ogawa (2000) et de Joaquin Pérez Azaústre (2015) recréent, autour de la figure du nageur, une ambiance solitaire. Le héros du récit de Joaquin Pérez Azaústre témoigne de cette solitude recherchée durant ses séances de natation : « il ne voit ni n'entend rien, si ce n'est le battement net et implacable de son propre cœur, dans cette obscurité débarrassée de l'écoute du monde. C'est une façon d'être seul : se réduire à une pulsation » (p. 13). Nombreux sont les nageurs à rendre compte de leur besoin ressenti de se retrouver seuls, face à eux-mêmes dans le bassin comme en témoigne les propos de Yann: «nager c'est quasiment de l'introspection, on est seul face à l'eau ». L'espace du bassin renvoie ainsi à la notion de territoire. Mais d'un «territoire personnel» (Singly, 2003) ${ }^{18}$, protégé des autres espaces traversés quotidiennement et dans lequel les nageurs souhaitent associer le moins de personnes possibles, fussent-elles de leur entourage. Si les nageurs ont le sentiment de se ressourcer grâce à leur séance de natation c'est précisément parce qu'elle assure une fonction de «mise à distance » d'avec les autres sphères de la vie sociale. La séance est ainsi décrite avec une insistance particulière sur l'adjectif possessif: «mon moment à moi », «mon cocon », «ma bulle», au sein desquels les nageurs viennent se ressourcer. L'instauration de ce moment privilégié de retour sur soi est notamment renforcée par l'acte du dépouillement vestimentaire. Revêtu de son uniforme de bain, le nageur matérialise, par le dépôt de ses vêtements au vestiaire, la mise à distance des statuts et des rôles qui lui incombent. Ainsi, le temps d'une séance, le nageur mis à nu n'est plus défini que par une unique dimension. Nager c'est alors se réduire et se protéger des agressions extérieures.

\subsection{Des nageurs structurés par l'habitude}

Si la natation est appréhendée par les nageurs comme un espace de compensation, comment parvient-elle à neutraliser les problématiques rencontrées? Il apparaît, qu'au-delà de la discipline proprement dite, le caractère répétitif et ritualiste de la pratique assure une fonction centrale dans la compensation de leurs difficultés physiques ou existentielles. En effet, il faut voir dans la réitération - parfois obsessionnelle - de leur venue au bassin un cadre, une structure, une source d'apaisement et

\footnotetext{
18 La notion de «territoire personnel » caractérise une sphère de la vie sociale dans laquelle l'individu se définit avant tout à titre personnel et non en référence à des statuts ou des positions occupées par ailleurs.
} 
d'équilibre venant neutraliser ces désagréments. Comme le souligne Jean Claude Kaufmann (Kaufmann, 2001), à la suite des travaux de Peter Berger et Thomas Luckmann (Berger, Luckmann, 2003 [1996]), les habitudes jouent un rôle essentiel dans la construction et le maintien du monde. À travers la routine qu'ils mettent en place, les nageurs donnent à voir un aspect bien différent du quotidien généralement associé $\mathrm{au}$ "train-train » et à l'ennui. La régularité de leur venue au bassin est, pour les nageurs, hautement porteuse de sens car elle constitue le socle de ce qui leur permet de tenir, de se maintenir ou d'avancer. La philosophe Carola Barbero rend compte, dans son traité sur la natation, de cette sécurité recherchée par les nageurs à travers l'exercice d'une pratique répétée "parce que dans sa répétitivité calme et obsessionnelle la natation engendre chez le praticien un sentiment de sécurité et un rythme intérieur qui l'envahit alors tout au long de son existence " (Barbero, 2016: 21). Le récit de Nathalia (43 ans, artiste) régulièrement traversée par l'angoisse et le doute révèle la dimension structurante de sa pratique réitérée : «c'est un cadre, une structure [...] ça structure de venir ici... ». Mais ces habitudes protectrices renvoient aussi, en creux, aux conséquences addictives que la natation est susceptible de produire chez les nageurs les plus assidus ${ }^{19}$. En effet, si la régularité de leur venue constitue une ascèse, une enveloppe protectrice, elle est aussi dépendance car ne pas nager renvoie à s'exposer au manque. Les périodes de vacances, de déplacements professionnels ou de fermeture d'établissement pour vidange sont particulièrement révélatrices des conduites addictives. Eloigné de son bassin le nageur élabore alors des stratégies - parfois complexes - pour s'enquérir d'un lieu alternatif où nager. Ils sont en effet nombreux à s'informer des horaires d'ouverture et de fermeture d'autres bassins situés à proximité, à se renseigner sur la présence d'une piscine non loin de leur destination. Et si, malgré leurs efforts ils ne parviennent pas à trouver un lieu pour nager, le retour au bassin s'accompagne souvent d'un commentaire ayant trait à cette privation.

\section{Conclusion}

Pour conclure, je souhaiterais ouvrir la réflexion sur une dimension de ce terrain «chez soi »: celle de la réciprocité de la relation d'enquête inhérente à ma position d’insider et ses conséquences dans la production des données recueillies. L'asymétrie, traditionnellement observée sur le terrain où chercheur et interlocuteur sont respectivement sujet et objet de la recherche est ici relative. Le statut de nageur qui nous rassemble, érode cette aspérité, plaçant au centre de la relation la réciprocité. En dépit

\footnotetext{
${ }^{19}$ Précision que le caractère addictif mentionné par certains nageurs n'est pas propre à la natation. On le retrouve dans d'autres sports d'endurance, notamment chez les coureurs à pieds (Segalen, 1994).
} 
de ma position de chercheur, connue de tous, je suis, au bassin, avant tout l'un des leurs, un nageur qui partage, avec eux, le goût de l'effort aquatique. La prévalence de cette dernière position sur celle du chercheur est favorisée par ma présence quotidienne qui estompe les enjeux de la recherche. Si la plupart de mes interlocuteurs m'ont dévoilé des pans de leur vie personnelle, voire de leur intimité, j'ai moi-même été amené à me raconter, non par stratégie, mais parce que la relation de confiance instaurée l'exigeait. Certaines de mes questions m'étaient alors naturellement renvoyées, auxquelles je me devais d'apporter des réponses. Ce renversement de position et la réflexivité qu'il induit m'a non seulement permis de soumettre à l'examen mes propres pratiques de nageur et les représentations associées mais a aussi rendu possible l'établissement d'une distance nécessaire au travail d'objectivation. L'épaisseur personnelle créée sur ce terrain et la réciprocité induite des relations nouées constitue l'ébauche d'une réflexion sur la distance. En effet, ce terrain «chez-soi » m'expose à deux principaux risques auxquels je dois me prémunir : l'« enclicage» (Olivier de Sardan, 1995) qui ne rendrait compte uniquement que des points de vue du groupe étudié et celui de la surinterprétation. Pour pallier à ces deux écueils je fais toujours part de mes résultats à d'autres nageurs extérieurs au bassin étudié, qui confortent, affinent ou révisent parfois certaines conclusions. C'est grâce à ce double mouvement articulé autour de la réciprocité de la relation d'enquête et de la confrontation des résultats recueillis à une altérité extérieure qu'il est possible de procéder au va-et-vient entre immersion et décentrement constitutif de la démarche ethnographique.

\section{Bibliographie}

Althabe G. (1990), "Ethnologie du contemporain et enquête de terrain », Terrain, vol. 14, p. 126-131.

BERGER P., LuCKMANN T. (2003 [1966]), La construction sociale de la réalité, Paris, Armand Colin.

Brosseau H. (2002), « L'eau et les soins. Avec les malades psychotiques chroniques », VST - Vie sociale et traitements, vol. 73, p. 39-49.

BARBERo C. (2016), L'arte di nuotare. Meditatzione sul nuoto, Genève, Il melangolo.

CHADI J. (2012), Contribution à l'étude de la sollicitation du métabolisme aérobie au cours d'un $100 \mathrm{~m}$ nage libre, thèse de doctorat, Poitiers, Université de Poitiers.

CORNIERE F. de (2015), Nageur du petit matin, Bègles, Le Castor Astral.

Kaufmann J.-C. (2001), Ego. Pour une sociologie de l'individu, Paris, Nathan.

Le Breton D. (2000), Eloge de la marche, Paris, Métailié.

OgaWa Y. (2000), La piscine, Paris, Acte Sud. 
OlIVIER DE SARDAN J.-P. (1995), « La politique du terrain. Sur la production des données en anthropologie », Enquête. Anthropologie, Histoire, Sociologie, vol. 1, p. 71-109.

Perez AzAústre J. (2015), Un nageur dans la ville, Paris, Seuil.

SCHIRRER M. (2007). «Espaces aquatiques urbains et mise en jeu corporelles, quelles affinités ?», Espace et Société, vol. 130, p. 151-167.

SCHWARTz O. (2011), « L'empirisme irréductible », in N. ANDERSON, Le Hobo, sociologie du sans-abri, Paris, Armand Colin, p. 335-384.

SEgalen M. (1994), Les enfants d'Achille et de Nike. Une ethnologie de la course à pied ordinaire, Paris, Métailié.

SEZNEC J-C. (2011), J'arrête de lutter avec mon corps. Votre thérapie par l'action, Paris, Presses universitaires de France.

SINGLY F. de (2003), «Intimité conjugale et intimité personnelle : à la recherche d'un équilibre entre deux exigences dans les sociétés modernes avancées », Sociologie et sociétés, vol. $35, \mathrm{n}^{\circ} 2$, p. 79-96.

Singly F. de, Martuccelli D. (2009), Les sociologies de l'individu, Paris, Armand Colin.

Souville M., Allegre B., Gentile S., Therme P. (2005), «Processus d'adaptation à une pratique sportive intensive: analyse exploratoire du discours de deux types de nageurs », Bulletin de psychologie, vol. 475, p. 81-90.

SCHNAPPER D. (2010), Une sociologue au Conseil constitutionnel, Paris, Gallimard.

TERRET T. (1992), Les défis du bain: formes de pratiques, modèles et résistances dans les processus de diffusion de la natation sportive, thèse de doctorat, Lyon, Université de Lyon 1.

Vigarello G. (1995), Le propre et le sale, Paris, Seuil.

VIVES B. (2008), Le goût du chlore, Paris, Casterman.

WACQUANT L. (2001), Corps et âme. Carnet ethnographique d'un apprenti boxeur, Marseille, Agone. 